\title{
COMPUTABLE ISOMORPHISM INVARIANTS FOR THE FUNDAMENTAL GROUP OF THE COMPLEMENT OF A PLANE PROJECTIVE CURVE
}

\author{
EDWARD M. ARNOLD
}

\begin{abstract}
The aim of this paper is to attach computable isomorphism invariants to the fundamental groups $\pi_{1}\left(\mathbf{P}^{2}-c\right)$ where $c$ is an irreducible plane projective curve. We use these invariants to distinguish certain of these groups. The vehicle used to obtain these invariants is the free differential calculus of R. Fox.
\end{abstract}

I. Introduction. As we shall see below, one can find a presentation for the group $\pi_{1}\left(\mathbf{P}^{2}-c\right)$. A presentation may not, however, tell us much about the group. In fact, it is an algorithmically unsolvable problem to decide whether two given presentations present isomorphic groups or not (cf. Rabin [5]). In view of this fact, it is useful to have computable isomorphism invariants for these groups. We will obtain these invariants below but, first, we need a presentation for $\pi_{1}\left(\mathbf{P}^{2}-c\right)$.

Let $c$ be an algebraic curve of degree $d$ in $\mathbf{P}^{2}$ (complex projective space). We want a presentation for the group $\pi_{1}\left(\mathbf{P}^{2}-c, e_{0}\right)$ where $e_{0}$ is any basepoint in $\mathbf{P}^{2}-c$. We briefly describe, following Cheniot [2], such a presentation.

There are only finitely many lines through $e_{0}$, namely those through singularities and tangent lines, which cut $c$ in fewer than $d$ points. Let $L_{g}$ be a line through $e_{0}$ which cuts $c$ in $d$ distinct points, and let $p$ be a point of $L_{g}$ different from $e_{0}$ and not on $c$. Let $L_{0}, L_{1}, \ldots, L_{m}$ be lines from $p$ which include the finitely many lines which cut $c$ in fewer than $d$ points. We also require $L_{i} \neq L_{g}, i=0,1, \ldots, m$. We let $b$ be a line through $e_{0}$ different from $L_{g}$. We set $A_{i}=b \cap L_{i}, 0 \leqslant i \leqslant m$, and $F_{i}=L_{g} \cap c, 1 \leqslant i \leqslant d$.

TheOREM 1. There are choices of loops $\eta_{j}$ in $b, 0 \leqslant j \leqslant m$, and $\gamma_{i}, 1 \leqslant i \leqslant$ $d$, in $L_{g}$ such that $\eta_{j}\left(\right.$ resp. $\left.\gamma_{i}\right)$ surrounds $A_{j}\left(\right.$ resp. $\left.F_{i}\right)$ respectively and exclusively and that the group $\pi_{1}\left(\mathbf{P}^{2}-c, e_{0}\right)$ can be presented as

$$
\begin{aligned}
\pi_{1}\left(\mathbf{P}^{2}-c, e_{0}\right)=\left\langle g_{1}, \ldots, g_{d} ; g_{i}=\phi_{i j}, g_{1} g_{2} \cdots g_{d}=1\right\rangle, & \\
1 & \leqslant i \leqslant d, 0 \leqslant i \leqslant m,
\end{aligned}
$$

where $g_{i}$ is represented by $\gamma_{i}$ and $\phi_{i j}$ is a word in the generators $g_{i}$ which is

Received by the editors May 17, 1976 and, in revised form, November 21, 1977.

AMS (MOS) subject classifications (1970). Primary 55A05, 55A10, 55A25, 14B05; Secondary 32B10, $20 \mathrm{CO5}$.

Key words and phrases. Isomorphism invariants, free differential calculus, plane projective cure, singular curve, fundamental group. 
represented by a loop which is obtained from $\gamma_{i}$ by a continuous deformation in the plane $L_{g}$ while the basepoint $L_{g} \cap b$ travels through $\eta_{j}$.

(For a proof, see Cheniot [2].)

We require the following results to make our algebraic apparatus work.

THEOREM 2. If $c$ is irreducible of degree $d$, then any two generators of $\pi_{1}\left(\mathbf{P}^{2}-c, e_{0}\right)$ are conjugate.

(For a proof too long to be presented here, see Theorem 3.3 of Arnold [1].)

THEOREM 3. If $c$ is irreducible of degree $d$, then $H_{1}\left(\mathbf{P}^{2}-c\right) \cong \mathbf{Z} / d \mathbf{Z}$.

Proof. Abelianize the above presentation. For details, see Theorem 3.5 of Arnold [1].

Theorems 2 and 3 as given here are Theorems 2 and 3 of Zariski [6].

II. Free differential calculus. In this section we describe an algebraic apparatus which produces isomorphism invariants for presentations of the groups $\pi_{1}\left(\mathbf{P}^{2}-c\right)$ where $c$ is an irreducible plane projective curve. The approach taken here is a modification of the one developed in Crowell and Fox [3] and Fox [4].

Let $x_{1}, x_{2}, \ldots, x_{n}, \ldots$ be a set of letters and let $F[x]=F\left[x_{1}, \ldots, x_{n}\right]$ be the free group on $x_{1}, \ldots, x_{n}$. We let $\pi=\left\langle x_{1}, \ldots, x_{n} ; R_{1}, \ldots, R_{k}\right\rangle$ be a presentation of $F[x] /\left\langle R_{1}, \ldots, R_{k}\right\rangle$ where $R_{1}, \ldots, R_{k} \in F[x]$ and $\langle\cdot\rangle$ denotes normal closure.

We denote by $\mathbf{Z}(G)$ the group ring over the integers of the group $G$. Given a group homomorphism $f: G \rightarrow H$ we can extend $f$ linearly to get a ring homomorphism (still denoted by $f$ ) $f: \mathbf{Z}(G) \rightarrow \mathbf{Z}(H)$. If $G \cong F[x] /$ $\left\langle R_{1}, \ldots, R_{m}\right\rangle$ we let $\gamma: F[x] \rightarrow G$ be the natural projection and a: $G \rightarrow$ $G / G^{1}$ the abelianizer where $G^{1}$ is the commutator subgroup of $G$. Henceforth we write $G / G^{1}=H$ and $F[x] /\left\langle R_{1}, \ldots, R_{m}\right\rangle=F[x] /\langle R\rangle$.

We define a derivation of type $(m, p)$ with $m, p \in \mathbf{Z}$ to be the linear extension of a multivalued function $D$ from $F[x]$ to $\mathbf{Z}(H)$ which satisfies:

$D_{1}$ : the set of values of $D\left(w_{1} \cdot w_{2}\right)=$ the set of values of $w_{1}^{p} D\left(w_{2}\right)+$ $D\left(w_{1}\right) w_{2}^{m}$.

$D_{\mathrm{II}}$ : the values of $u^{-p} D(u) u^{-m} ; p, m \in \mathbf{Z}$, are values of $D\left(u^{-1}\right)$.

REMARKS. The function $D$ can be multivalued. It can happen that $w_{1} \cdot w_{2}=$ $w_{1} \cdot x_{i}^{-\varepsilon} \cdot x_{i}^{\varepsilon} \cdot w_{2}, \varepsilon= \pm 1$, while $D\left(w_{1} \cdot w_{2}\right) \neq D\left[\left(w_{1} x_{i}^{-\varepsilon}\right) \cdot\left(x_{i}^{\varepsilon} w_{2}\right)\right]$ computed by the product rule. A short computation shows, however, that the map $w \mapsto \operatorname{ar} D(w)$ is single valued. Since we are only interested in the values $\operatorname{ar} D(w)$ and since we may simply reduce a word such as $w_{1} x_{i}^{-\varepsilon} x_{i}^{\varepsilon} w_{2}$ to $w_{1} \cdot w_{2}$ we never encounter multiple values for $D$. The general multiple valuedness of $D$ does force us to state $D_{\mathrm{II}}$ as a condition rather than derive it from $D_{\mathrm{I}}$.

The derivations we shall need are described in the following lemma.

LEMMA 4. For each $x_{j}, 1 \leqslant j \leqslant n$, there is a unique derivation $D_{j}$ satisfying $D_{\mathrm{I}}$ and $D_{\mathrm{II}}$ for fixed $p$ and $m$, such that 
(a)

$$
\begin{aligned}
D_{j}\left(x_{i}\right) & =\delta_{i j}=0 \quad \text { if } i \neq j, \\
& =1 \quad \text { if } i=j
\end{aligned}
$$

(b) a $\gamma D$ is single valued.

Proof. It is not hard to see that there is a unique derivation $D_{j}$ with $D_{j}\left(x_{i}\right)=\delta_{i j}$ which satisfies $D_{\mathrm{I}}$. It follows from the rule $D_{\mathrm{I}}$ that $D(1)=0$. Using this we see that $D\left(u \cdot u^{-1}\right)=0$ which implies $D_{\mathrm{II}}$. A computation shows that $\operatorname{ar} D\left(w_{1} w_{2}\right)=\mathfrak{a} \gamma D\left(w_{1} x_{j}^{-\varepsilon} x_{j}^{e} w_{2}\right)$ from which (b) follows.

Let $\pi=\left\langle x_{1}, \ldots, x_{n} ; R_{1}, \ldots, R_{m}\right\rangle$ be a presentation for $G$. The Alexander matrix $A(\pi)$ for the presentation $\pi$ is the $n \times m$ matrix whose $(i, j)$ th entry is $\mathfrak{a} \gamma D_{j}\left(x_{i}\right) \in \mathbf{Z}(H)$. Here $\gamma: \mathbf{Z}(F[x] /\langle R\rangle) \rightarrow \mathbf{Z}(G)$ and $\mathfrak{a}: \mathbf{Z}(G) \rightarrow \mathbf{Z}(H)$. Since $\mathbf{Z}(H)$ is commutative we can define the following elementary ideals of $\pi$.

$\varepsilon_{k}(\pi)=$ the ideal in $\mathbf{Z}(H)$ generated by the determinants of

$$
\begin{aligned}
& \text { the }(n-k) \times(n-k) \text { minors of } A(\pi) \text { if } 0<n-k \leqslant m, \\
= & 0 \text { if } n-k>m, \\
= & \mathbf{Z}(H) \text { if } n-k \leqslant 0 .
\end{aligned}
$$

The following theorem shows that the elementary ideals are isomorphism invariants.

THEOREM 5. Let $\pi$ and $\psi$ be presentations of the same group. Then $\mathcal{E}^{k}(\pi)=$ $\xi^{k}(\psi)$ for all $k$.

Proof. The proof which is a slight generalization of a similar theorem of $\mathbf{R}$. Fox can be found in Theorem 4.1 of Arnold [1].

In order to apply the free differential calculus to the case $G=\pi_{1}\left(\mathbf{P}^{2}-c\right)$ the group ring $\mathbf{Z}(H)$ should be a reasonable ring in which to work and the Alexander matrix should be effectively computable from the matrix of derivatives $\left[D_{j}\left(R_{i}\right)\right]$ (with $R_{i}$ reduced to avoid multiple values). First of all, when $G=\pi_{1}\left(\mathbf{P}^{2}-c\right), H$ is the cyclic group $\mathbf{Z} / d \mathbf{Z}$. Hence the group ring $\mathbf{Z}(H)$ is the polynomial ring $\mathbf{Z}[t]$ reduced modulo $d$. The desirable condition on the Alexander matrix is guaranteed by the following lemma.

LEMMA 6. Let $c$ be an irreducible plane projective curve. The homomorphism $a \circ \gamma: \mathbf{Z}(F[x]) \rightarrow \mathbf{Z}(H)$ carries each generator $g_{i}, 1 \leqslant i \leqslant d$, of $\pi_{1}\left(\mathbf{P}^{2}-c\right)$ to a single generator $t$ of $H=H_{1}\left(\mathbf{P}^{2}-c\right)$.

Proof. Let $g_{0}$ be any fixed generator of $\pi_{1}\left(\mathbf{P}^{2}-c\right)$ and let $g$ be any other generator. According to Theorem $2, g$ and $g_{0}$ are conjugates. Hence $g_{0}=$ $h g h^{-1}$ for some $h \in \pi_{1}\left(\mathbf{P}^{2}-c\right)$. We can therefore write

$$
\mathfrak{a} \circ \gamma(g)=\mathfrak{a} \circ \gamma\left(h \bar{g}_{0}^{1} h\right)=\mathfrak{a} \circ \gamma\left(h^{-1}\right) \mathfrak{a} \circ \gamma\left(g_{0}\right) \mathfrak{a} \circ \gamma(h)=\mathfrak{a} \circ \gamma\left(g_{0}\right) .
$$

We choose $t=a \circ \gamma\left(g_{0}\right)$.

We can now state a necessary condition for $\pi_{1}\left(\mathbf{P}^{2}-c\right)$ to be abelian.

THEOREM 7. Let $c$ be an irreducible plane projective curve of degree $d$ and let $D_{t}$ be as described in Lemma 4. In order for $\pi_{1}\left(\mathbf{P}^{2}-c\right)$ to be abelian it is 
necessary that

$$
\begin{aligned}
& \mathcal{E}_{k}(c)=\mathbf{Z}(\mathbf{Z} / d \mathbf{Z}) \text { for } k \geqslant 1 \text { and } \\
& \mathcal{E}_{0}(c)=\left(D_{t}\left(t^{d}\right)\right) \subset \mathbf{Z}(\mathbf{Z} / d \mathbf{Z}) .
\end{aligned}
$$

Proof. If $\pi_{1}\left(\mathbf{P}^{2}-c\right)$ is abelian then it is isomorphic to $\mathbf{Z} / d \mathbf{Z}$ by Theorem 3. Since $\mathcal{E}_{k}(c)$ are isomorphism invariants, it suffices to show the result for $\pi=\left\langle t ; t^{d}\right\rangle$. The result now follows from the definition of the elementary ideals.

III. Examples and results. We let $A^{(n, m)}$ and $\mathcal{E}_{k}^{(n, m)}$ denote the Alexander matrix and elementary ideals corresponding to the derivations $D_{j}^{(n, m)}$ which satisfy:

$$
\begin{aligned}
& D_{j}^{(n, m)}(u \cdot v)=u^{n} D_{j}^{(n, m)}(v)+D^{(n, m)}(u) v^{m} \text { and } \\
& D_{j}^{(n, m)}\left(u^{-1}\right)=-u^{-n} D_{j}^{(n, m)}(u) \cdot u^{-m} .
\end{aligned}
$$

EXAMPLE 1. We let $c_{1}$ be an irreducible sextic with six cusps on a conic. In this case it can be shown (Zariski [6]) that $\pi_{1}\left(\mathbf{P}^{2}-c_{1}\right)=\left\langle g_{1}, g_{2} ; g_{1}^{2}, g_{2}^{3}\right\rangle$. We let $c_{2}$ be an irreducible nonsingular sextic. A theorem of Zariski says that $\pi_{1}\left(\mathbf{P}^{2}-c_{2}\right) \cong \mathbf{Z} / 6 \mathbf{Z}$. The two groups $\pi_{1}\left(\mathbf{P}^{2}-c_{1}\right)$ and $\pi_{1}\left(\mathbf{P}^{2}-c_{2}\right)$ are distinguishable by the elementary ideals.

THEOREM 8. Let $c_{1}$ and $c_{2}$ be as above. The elementary ideal $\mathcal{E}_{0}(1,2)$ distinguishes $\pi_{1}\left(\mathbf{P}^{2}-c_{1}\right)$ and $\pi_{1}\left(\mathbf{P}^{2}-c_{2}\right)$.

Proof. Since we are interested in the values $a \gamma D(w)$ and $a \gamma D$ is single valued for a derivation $D$, we may use the product rule $D(u v)=u D v+D u$. $v^{2}$ and $D\left(u^{-1}\right)=-u^{-1} D(u) u^{-2}$. We get

$$
\begin{aligned}
& D_{1}\left(g_{1}^{2}\right)=g_{1}+g_{1}^{2}, \quad D_{2}\left(g_{2}^{3}\right)=g_{2}\left(g_{2}+g_{2}^{1}\right)+g_{2}^{4}=g_{2}^{2}+g_{2}^{3}+g_{2}^{4}, \\
& A^{(1,2)}\left(c_{1}\right)=\left(\begin{array}{cc}
t+t^{2} & 0 \\
0 & t^{2}+t^{3}+t^{4}
\end{array}\right), \\
& \mathcal{E}_{0}^{(1,2)}\left(c_{1}\right)=\left(1+t^{3}+2 t^{4}+2 t^{5}\right), \\
& \mathcal{E}_{1}^{(1,2)}\left(c_{1}\right)=\left(t+t^{2}, t^{2}+t^{3}+t^{4}\right)=\mathbf{Z}(\mathbf{Z} / 6 \mathbf{Z}), \\
& \mathcal{E}_{k}^{(1,2)}\left(c_{1}\right)=\mathbf{Z}(\mathbf{Z} / 6 \mathbf{Z}), \quad k>1 .
\end{aligned}
$$

For the curve $c_{2}$ with $\pi_{1}\left(\mathbf{P}^{2}-c_{2}\right)=\left\langle g ; g^{6}\right\rangle$

$$
\begin{aligned}
& \mathcal{E}_{0}^{(1,2)}\left(c_{2}\right)=\left(1+t+t^{2}+t^{3}+t^{4}+t^{5}\right), \\
& \mathcal{E}_{k}^{(1,2)}\left(c_{2}\right)=\mathbf{Z}(\mathbf{Z} / 6 \mathbf{Z}), \quad k \geqslant 1 .
\end{aligned}
$$

In view of the above computation it suffices to show that $1+t^{3}+2 t^{4}+t^{5}$ is not divisible by $1+t+t^{2}+t^{3}+t^{4}+t^{5}$ in the ring $\mathbf{Z}(\mathrm{Z} / 6 \mathbf{Z})$. For this, suppose that there are integers $a, b, c, d, e$ and $f$ such that

$$
\begin{aligned}
\left(a+b t+c t^{2}+d t^{3}+e t^{4}+f t^{5}\right) \cdot\left(1+t+t^{2}+t^{3}+t^{4}+t^{5}\right) \\
=1+t^{3}+2 t^{4}+2 t^{5}
\end{aligned}
$$

This implies the contradictory statements $a+b+c+d+e+f=0$ and $a+b+c+d+e+f=1$. The last assertion of the theorem is clear since if 
$\pi_{1}\left(\mathbf{P}^{2}-c_{1}\right)$ were abelian we would have $\pi_{1}\left(\mathbf{P}^{2}-c_{1}\right) \cong H_{1}\left(\mathbf{P}^{2}-c_{1}\right) \cong$ $\mathbf{Z}(\mathbf{Z} / 6 \mathbf{Z}) \cong \pi_{1}\left(\mathbf{P}^{2}-c_{2}\right)$.

REMARK. Computations show that $\mathcal{E}_{k}^{(1,0)}, \mathcal{E}_{k}^{(1,1)}$ and $\mathcal{E}_{k}^{(-1,1)}$ fail to distinguish the groups $\pi_{1}\left(\mathbf{P}^{2}-c_{1}\right)$ and $\pi_{1}\left(\mathbf{P}^{2}-c_{2}\right)$.

EXAMPLE 2. $c_{1}$ is an irreducible three cuspidal quartic and $c_{2}$ is an irreducible nonsingular quartic. In this case $H=\mathbf{Z} / 4 \mathrm{Z}$ and it can be shown that $\pi_{1}\left(\mathbf{P}^{2}-c_{1}\right)=\left\langle g_{1}, g_{2} ; g_{1}^{2} g_{2}^{-2}, g_{1}^{4},\left(g_{1} g_{2}\right)^{3} g_{1}^{-2}\right\rangle$ (cf. Zariski [6]). We have the following result.

THEOREM 9. There exists projective plane curves of the same degree, namely the three cuspidal quartic and the nonsingular quartic, which cannot be distinguished by $\mathcal{E}_{k}^{(n, m)}$ for any integers $n, m$ and $k$.

Proof. Since $H=\mathbf{Z} /$ ' $\mathbf{Z}$, and $\mathbf{Z}(H)$ is commutative, we get the following equalities.

$$
\begin{aligned}
& \operatorname{ar} D^{(1,0)}(w)=\operatorname{ar} D^{(0,1)}(w)=\operatorname{a} \gamma D^{(0,3)}(w)=\operatorname{ar} D^{(3,0)}(w), \\
& \mathfrak{a} \gamma D^{(1,-1)}(w)=\mathfrak{a} \gamma D^{(-1,1)}(w)=\mathfrak{a} \gamma D^{(1,3)}(w)=\mathfrak{a} \gamma D^{(3,1)}(w), \\
& \operatorname{a} \gamma D^{(1,2)}(w)=a \gamma D^{(2,1)}(w)=\operatorname{ar} D^{(2,3)}(w)=\mathfrak{a} \gamma D^{(3,2)}(w) \\
& \text { for all } w \in F\left[g_{1}, g_{2}\right] \text {. }
\end{aligned}
$$

Hence it is enough to show that the three cuspidal quartic and the nonsingular quartic cannot be distinguished by $\mathcal{E}_{k}^{(1,0)}, \mathcal{E}_{k}^{(1,-1)}$ and $\mathcal{E}_{k}^{(1,2)}$. An inspection of the list of elementary ideals below shows that we need only verify that $\left(t^{3}+t\right)=\left(4 t^{2}+4 t, 6 t^{2}+6,3+t+3 t^{2}+t^{3}\right)$ and $\mathbf{Z}(\mathrm{Z} / 4 \mathrm{Z})=(1+t, 1+t$ $\left.+t^{2}+t^{3}, 1-t+t^{2}, 2 t+t^{3}\right)$. These facts follow from straightforward computations.

List of elementary ideals.

$$
\begin{aligned}
\mathcal{E}_{k}^{(1,0)}\left(c_{1}\right) & =\left(1+t+t^{2}+t^{3}\right), \quad k=0, \\
& =\left(1+t, 1+t+t^{2}+t^{3}, 1-t+t^{2}, 2 t+t^{3}\right), \quad k=1 \\
& =\mathbf{Z}(\mathbf{Z} / 4 \mathbf{Z}), \quad k>1 ; \\
\mathcal{E}_{k}^{(-1,1)}\left(c_{1}\right) & =\left(4 t^{2}+4 t, 6 t^{2}+6,3+t+3 t^{2}+t^{3}\right), \quad k=0 \\
& =\mathbf{Z}(\mathbf{Z} / 4 \mathbf{Z}), \quad k \geqslant 1 ; \\
\mathcal{E}_{k}^{(1,2)}\left(c_{1}\right) & =\left(1+t+t^{2}+t^{3}\right), \quad k=0, \quad k=1 \\
& =\left(1+t, 1+t+t^{2}+t^{3}, 1-t+t^{2}, 2 t+t^{3}\right), \quad k=1 ; \\
& =\mathbf{Z}(\mathbf{Z} / 4 \mathbf{Z}), \quad k>1 ; \\
\mathcal{E}_{k}^{(1,0)}\left(c_{2}\right) & =\left(1+t+t^{2}+t^{3}\right), \quad k=0, \\
& =\mathbf{Z}(\mathbf{Z} / 4 \mathbf{Z}), \quad k \geqslant 1 ; \\
\mathcal{E}_{k}^{(1,-1)}\left(c_{2}\right) & =\left(t^{3}+t\right), \quad k=0, \\
& =\mathbf{Z}(\mathbf{Z} / 4 \mathbf{Z}), \quad k \geqslant 1 ; \\
\mathcal{E}_{k}^{(1,2)}\left(c_{2}\right) & =\left(1+t+t^{2}+t^{3}\right), \quad k=0 \\
& =\mathbf{Z}(\mathbf{Z} / 4 \mathbf{Z}), \quad k \geqslant 1 .
\end{aligned}
$$


REMARKS. The above procedure can be applied to presentations of the local groups $\pi_{1}\left(\bar{\Delta}_{\varepsilon}(0) \cap C^{2}-c\right)$ where $\bar{\Delta}_{\varepsilon}(0)$ is a small closed polydisc about $0 \in C^{2}$, and $c$ is an algebraic curve with a singularity at 0 . We mention two of these results. (For details, see Arnold [1].)

1. There exist algebraic singularities with the same multiplicities whose local groups are indistinguishable by $\mathcal{E}_{k}^{(n, m)}$ for all $n, m$ and $k$. Examples are the simple cusp and the cubo-quadratic cusp. ( $x=t^{3}, y=t^{5}$ near the origin.)

2. The local groups $\pi_{1}\left(\bar{\Delta}_{\varepsilon}(0) \cap C^{2}-c\right)$ of the cubical cusp and the cubo-quadratic cusp are not isomorphic. They are distinguished by $\mathcal{E}_{1}^{(1,0)}$.

We also remark that the coarseness of the elementary ideals as reflected in Theorem 9 and Remark 2 above is not surprising in view of the coarseness of the elementary ideals for knots in $R^{3}$. For more discussion on the coarseness of the elementary ideals, see Arnold [1].

\section{REFERENCES}

1. E. M. Arnold, Computable isomorphism invariants for the fundamental group of the complement of a plane projective curve, Ph.D. Thesis, Univ. of Washington, Spokane, 1975.

2. D. Cheniot, Le théorème de Van Kampen sur le group fondamental du complémentaire d'une courbe algébrique projective plane, Seminaire Norguet, Lecture Notes in Math., vol. 409, Springer-Verlag, New York, 1974.

3. R. H. Crowell and R. H. Fox, Introduction to knot theory, Ginn, Boston, Mass., 1962.

4. R. H. Fox, Free differential calculus. II. The isomorphism problem of groups, Ann. of Math. (2) 59 (1954), 196-210.

5. M. O. Rabin, Recursive unsolvability in group theoretic problems, Ann. of Math. (2) 67 (1958), 172-194.

6. O. Zariski, On the existence of algebraic functions of two variables possessing a given branch curve, Amer. J. Math. 51 (1929), 305-328.

Department of Mathematics, Whitworth College, Spokane, Washington 99251

Current address: Battelle Pacific Northwest Laboratories, Battelle Boulevard, Richland, Washington 93352 